\title{
Enhancing DInSAR capabilities for landslide monitoring by applying GIS-based multicriteria filtering analysis.
}

\author{
Fasil Beyene, Steffen Knospe, Wolfgang Busch \\ Clausthal University of Technology, Institute of Geotechnical Engineering and Mine Surveying, \\ Erzstrasse 18, D-38678 Clausthal-Zellerfeld, Germany, \\ Email: fasil.beyene@tu-clausthal.de
}

KEY WORDS: Landslide monitoring, DInSAR, GIS multicriteria analysis

\begin{abstract}
:
Landslide detection and monitoring remain difficult with conventional differential radar interferometry (DInSAR) because most pixels of radar interferograms around landslides are affected by different error sources. These are mainly related to the nature of high radar viewing angles and related spatial distortions (such as overlays and shadows), temporal decorrelations owing to vegetation cover, and speed and direction of target sliding masses. On the other hand, GIS can be used to integrate spatial datasets obtained from many sources (including radar and non-radar sources). In this paper, a GRID data model is proposed to integrate deformation data derived from DInSAR processing with other radar origin data (coherence, layover and shadow, slope and aspect, local incidence angle) and external datasets collected from field study of landslide sites and other sources (geology, geomorphology, hydrology). After coordinate transformation and merging of data, candidate landslide representing pixels of high quality radar signals were filtered out by applying a GIS based multicriteria filtering analysis (GIS-MCFA), which excludes grid points in areas of shadow and overlay, low coherence, non-detectable and non-landslide deformations, and other possible sources of errors from the DInSAR data processing. At the end, the results obtained from GIS-MCFA have been verified by using the external datasets (existing landslide sites collected from fieldworks, geological and geomorphologic maps, rainfall data etc.).
\end{abstract}

\section{INTRODUCTION}

SAR data acquired by recent high resolution radar satellites are increasingly being used in slope stability assessment and monitoring of landslide (Singhroy 2005, Ferretti et al. 2005). The different Line-of-Sight (LOS) imaging capability of TSX/TDX and combining data from different tracks have also increased its suitability for slope instability assessment and monitoring of landslides, especially in mountainous terrains. However, owing to the nature of high radar viewing angles and related spatial distortions (such as overlays and shadows), temporal decorrelations due to vegetation cover, and speed and direction of target sliding masses, monitoring of landslides using conventional DInSAR methods remains difficult.

\section{SAR DATA ACQUISITION}

The area selected for this study, the central part of Dessie town and its environs, Ethiopia, is bounded by steep mountain ranges that run from North-South direction. To match the SAR data acquisition with the topography of the area, the appropriate look angles were considered in both ascending $\left(\sim 25^{\circ}\right)$ and descending $\left(\sim 28^{\circ}\right)$ SAR acquisition geometries. In such a way that as satellite sensors acquire data over the ascending tracks looking towards the east direction, the slope instability or deformation phenomena mainly along the hillsides of Tossa fault scarp will be effectively monitored. On the other hand, the slope failures along the Azwa valley slope facets will be detected by satellite in the descending orbits, as it looks towards the west.

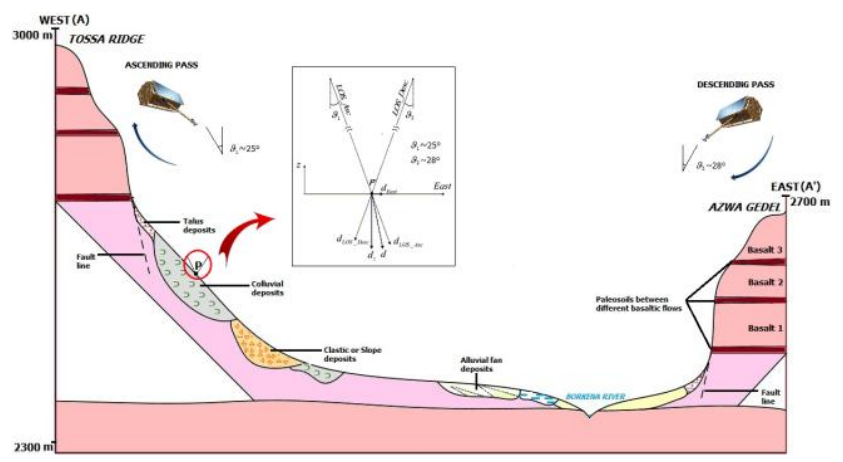

Figure 1. The selected image acquisition geometries in relation to the local topography and target landslide body

\section{METHODOLOGY}

The proposed work procedure (Figure 2.) in this research work attempts to enhance the DInSAR capabilities for landslide 
monitoring by systematically extracting landslide representative candidate deformation points (DPs) or excluding DPs which are affected by noise sources or non-relevant deformations with respect to landslide phenomena. To achieve this, first regularly spaced grid points are created in map coordinate system encompassing the whole area of the raster datasets. The displacement maps, representing the LOS displacement phase component, in radar coordinate systems are projected to the map coordinate system so that extraction of raster point and data integration with the other GIS datasets (non-radar or external data sources (GPS measurements, geological, structural, hydrological data, soil moisture etc.) can be plausible. To achieve this, a GIS based multicriteria filtering analysis (GIS-MCFA) is used to filter out candidate landslide pixels or deformation points (CL-Ps/CL-DPs). Appropriate criteria have been applied to exclude the possible error sources of DInSAR and unwanted deformations recorded by the radar sensors (such as uplifting or upward motions). Finally, the result of the GIS-MCFA have been further analysed by using time-series curves and interpreted by overlaying with external datasets.

The original SAR data required for this analysis was processed in radar coordinate system by using conventional 2-pass DInSAR method. Hence, for the purpose of GIS-MCFA, all the radar origin datasets have been transformed from radar rangeDoppler coordinates to geographic coordinate system. The general work procedures followed to process the GIS-MCFA datasets are indicated in the workflow (Figure2.).

A GRID-DP model is used to represent the pixel location and values of DInSAR LOS displacement maps as a grid of deformation points (DPs). In addition to displacement maps, other radar derived maps (such as coherence, slope/aspect, overlay/shadow etc.) and external source datasets (landslide site, GPS, geology and geomorphology, hydrological, land use and land cover etc.) are converted into a grid of points. These radar and non-radar origin point datasets have the same spatial extent, so that they can be merged into a single vector file with many attributes.

Every pixel in the radar images can be converted into point features by converting the midpoint of the raster pixel. For the sake of the GIS-MCFA, all the necessary project data, the various output map layers of interferometric pairs and other non-radar external map layers, have been converted into point features and merged into a single data file which makes the GIS-MCFA data analysis easier. In this sense, GRID-DP model can be consider as an extraction tool to extract the valuable numerical information from radar, and external vector and raster data sources.

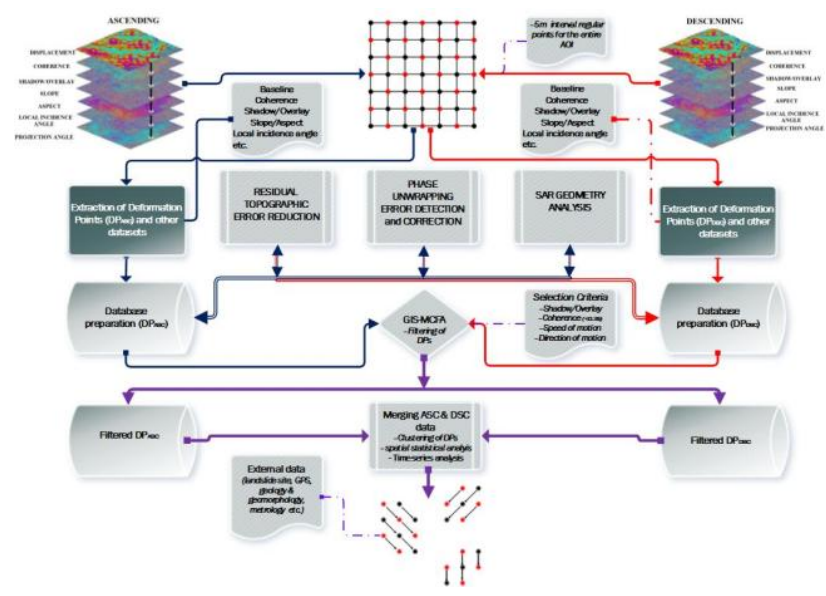

Figure 2. The workflow of data analysis.

The compaction of all the necessary raster datasets into a single point dataset has the following main advantages.

- Comparison between deformation values extracted from many raster maps and spotting phase unwrapping error/s incurring pixel/s in spreadsheet formats is easier.

- Relatively less data storage requirement is fulfilled. As many raster data layers are compacted into a single vector file format, the size of the resulting data will largely be reduced, and the data analysis, which would have been cumbersome to do it in raster format, can be practical.

Furthermore, it allows us to work flexibly and comfortable in other common spatial data processing software.

\subsection{GIS-based multicriteria filtering analysis}

The main goal of GIS based multi-criteria analysis (GISMCFA) is to filter out candidate landslide pixels or deformation points (CL-Ps/CL-DPs) from the line-of-sight displacement values of radar images extracted in grid format by converting each radar displacement image pixel into DPs and setting relevant filtering criteria. In order to achieve that the extracted LOS displacement values were merged with other helpful parameters like coherence and geometric parameters (slope/aspect, layover and shadow, and local incidence angle) identified as per the criteria selection assessment done, and then 
classified into overlay/shadow and/or normal (slant range resolution), coherent and/or low-coherent, stable and/or nonstable, uplifting and/or subsiding pixels so as to filter out the landslide areas (Error! Reference source not found.1.).

Considering the phase difference between two radar images acquired over the same area, the LOS displacement values calculated from these images represent the components of the phase related to geometric displacements of objects and other error sources due to (residual topography, atmosphere disturbances, orb it and phase decorrelation noise). In other words, the displacement values in the radar images consists of areas covered by overlay/shadow, phase noise, coherent and/or low-coherent, stable and/or non-stable, uplifting and/or subsiding pixels. Accordingly, the GRID-DPs data are broadly classified by using parameters that control the deformation nature (based on the speed and direction of motion) and the error sources of the data.

\subsubsection{Error control parameters:}

These parameters are set to exclude the data gaps related to the geometric distortion in the high slope or mountainous areas (such as overlay/shadow and foreshortening) and phase errors as a result of low coherence.

\subsubsection{Deformation control parameters}

SAR sensors have limitations in monitoring landslide motion above a certain speed and direction of motion due the wavelength range they operate and side-looking nature of radar sensors. Thus, these controlling parameters are re-classified as: slow motions

Direction of Motion: non-LOS (non-line-ofsight) motion, upwards and downwards motions

\subsubsection{DP filtering criteria}

In order to filter out the optimal CL-Ps/CL-DPs of high quality radar signals that best represent the landslide sites from a grid model, the following six filtering criteria were considered. The ASC and DSC orbit data were treated separately during the multicriteria filtering analysis.

\subsubsection{Shadow and Overlay}

Due to side-looking nature of SAR sensors, spatial distortions (such as shadow and overlay) occur in the radar images. These distortions appear to be worse in areas of high topographic relief where slope instabilities often occur. As illustrated in (Figure 2.) below, areas between points $\mathrm{J}$ and $\mathrm{N}$ cannot be illuminated by the radar beam. As a result, SAR resolution cells from $\mathrm{J}$ to $\mathrm{N}$ do not record any radar signal from the ground and they appear dark on the image. Hence, DPs falling in the shadow regions should be eliminated from grid.

On the other side, DPs that are located at increasing ground range positions can be imaged in reverse order by the SAR system (as depicted by letters F, G, H, and I). In addition, they are recorded in the same SAR resolution cells as DPs located on $\mathrm{D}$ and $\mathrm{E}$, which belong to a different area on the ground. These DPs are directly or indirectly affected by layover and effect of overlay. Similarly, DPs that fall in overlay zone should be eliminated from grid.

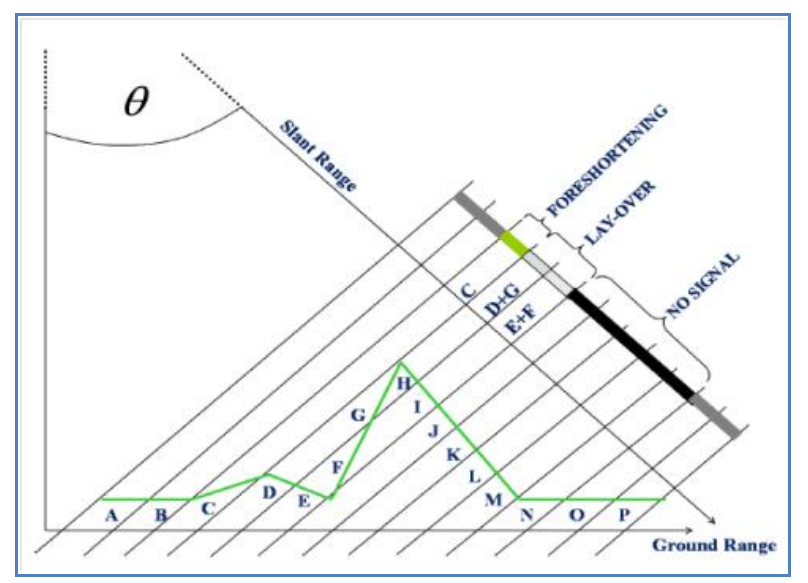

Figure 2. The different geometric distortions in(such as overlay and shadow) explained as the $3 \mathrm{D}$ image is projected onto a $2 \mathrm{D}$ plane (Ferretti et al. 2007).

The overlay/shadow map used to exclude DPs was generated by using a gc_map algorithm in gamma software. However, it is also possible to calculate the part of a given area affected by overlay/shadow from digital elevation information, more specifically if we know the slope and look angle or depression angle of the radar antenna. For this project, look angles $\boldsymbol{\theta}$ are approximately $25^{\circ}$ and $28^{\circ}$ for radar images acquired from ascending and descending orbits respectively. Hence, areas will be affected by overlays or its effects if the slope angle (u) is greater than look angle $\boldsymbol{\theta}$. Similarly, shadows are a result of very steep slope and occur when the slope angle $(\mathbf{u})$ is greater 
than depression angle, $\boldsymbol{\alpha}$, irrespective of the satellite orbit direction in both cases (Colesanti and Wasowski 2006). The depression angle is complementary to the look angle.

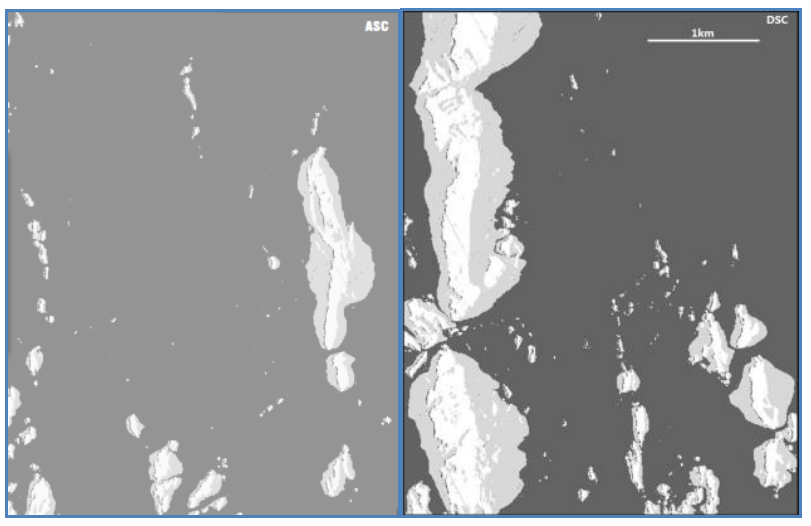

Figure 1. The overlay/shadow maps of the project area showing the areas affected by different distortion effects in ascending (left) and descending (right).

\subsubsection{Coherence}

The presence of several scatterers within each SAR resolution cell generates speckle effects to SAR images. Pixels around these areas are affected by different kinds of error sources, and often cause also difficulties in phase unwrapping process. Hence, DPs falling in these areas should be removed from the GRID-DP. The coherent map used to identify low-coherent DPs from the grid and DPs with a coherent value less than 0.35 were removed.

\subsubsection{Geology and geomorphology}

The main moving masses in the area of interest are usually consists of secondary deposits or sediments which are formed due to the recent mass wasting processes (Tenalem and Barbieri, 2005, Fubelli et al. 2008). However, there are some known locations which are identified as non-susceptible to the occurrence of landslides due to a unique geological geomorphological formation.

Though the exposed basalts within the study area have undergone variable degrees of chemical and physical weathering, and are exposed to rock falls and topples (especially around the fault escarpments of Tossa and Azwa Gedel) (Tenalem and Barbieri 2005, Fubelli et al. 2008), but they are not expected to experience slow moving type of motion which can be monitored by DInSAR methods. Hence, for this particular project, it is logical to exclude these areas from the DInSAR based monitoring.

\subsubsection{Extremely fast motion}

SAR images acquired by radar satellites can only record motions at scale of spatial and temporal resolution of the imaging sensor (Massonnet and Feigl 1998). Assuming that topographic fringes are properly removed from interferograms using external source digital elevation model, each fringe corresponds to change in range of half of the radar wavelength $(\boldsymbol{\lambda} / 2)$ where $\boldsymbol{\lambda}=31 \mathrm{~mm}$ and $\mathrm{t}=11$ days for TSX/TDX. The phase measurements are modulo- $2 \pi_{v}$ which corresponds to $\lambda / 2$ and the time span covered by interferogram exceeds $\lambda / 4$ when such kind of fast motions occurred. Consequently, the interferograms remain incoherent because the detectable motion range is exceeded. As result, DInSAR experience problem in detection of such kind of fast ground deformations due to loss of coherence in the interferogram.

The mountain flanks having very steep slope angle greater than $45^{\circ}$ usually experience very to extremely fast moving landslides such as rock fall or avalanche, topple. Nevertheless, these kind of fast movements are difficult to detect by currently available DInSAR technology (Colesanti and Wasowski 2006).

\subsubsection{Extremely slow motion}

"Extremely slow motion" are defined for this particular GISMCFA purpose as stable areas or extremely slow moving landslides that are not our interest of highest importance. This is, first, partly because of a little threat on human-life as they give us time to react against them or the relative minimal risk they pose on infrastructure.

This is, in fact, a very controversial parameter as there is a possibly of reactivation of landslide motion after a certain dormant period following anomalous rainfalls or sudden shakes. However, this selection criterion remains very important because it helps to differentiate the stable and non-stable DPs at least for a single and/or if we are lucky for many more successive acquisitions. Thus, it is logical to use this parameter to control the nature of deformation for these specific acquisitions. Consequently, landslide suspected areas were 
identified and separated from stable places by setting a controlling threshold value for DPs in each scene. As per the critical observation of the processed data, the assumed controlling threshold value for this criterion was $1 \mathrm{~mm}$ per each 11 days acquisition period, the shortest revisit for the datasets available. In this context, if the recorded DPs values are found to be below $1 \mathrm{~mm}$, then they will be assigned to extremely slow moving or stable DPs and extracted from the GRID Model.

\subsubsection{Non-los motion}

SAR satellites are only able to measure movements in the lineof-sight (LOS) direction. The registered displacement along LOS could only be the same as that of the real displacement (i.e. $100 \%$ preserved) when the displacement is parallel to the LOS. In the contrary, the detectable motion could reduce to $0 \%$ if the displacement direction is parallel to the azimuth direction (flight direction of the satellite) (Metternicht et al. 2005). This implies that SAR systems have limitations in measuring movements along azimuth or close to $\mathrm{N}^{-} \mathrm{S}$ direction (Error!

\section{Reference source not found.).}

As illustrated in (Figure 4.), the percentage of detectable movement by SAR sensor decreases as the orientation of the slope dip direction closes to the direction of flight. For the sake of simplicity and use in MCFA, it is logical to assume that the amount of motion to be measured from slopes facing azimuth directions (some $30^{\circ} \mathrm{N}-\mathrm{S}$ from azimuth direction) are too small to be measurable or negligible by the SAR sensor. For example, for the TSX/TDX ascending data, the N-S facing slopes are identified as $0^{\circ}<\mathrm{v}<7^{\circ}, 337^{\circ}<\mathrm{v}<360^{\circ}$ (north oriented slopes) and $157^{\circ}<\mathrm{v}<187^{\circ}$ (south facing slopes), where $\mathrm{v}$ is the aspect angle of the slope. Consequently, DPs falling along these zones should be eliminated from the GRID-DP.

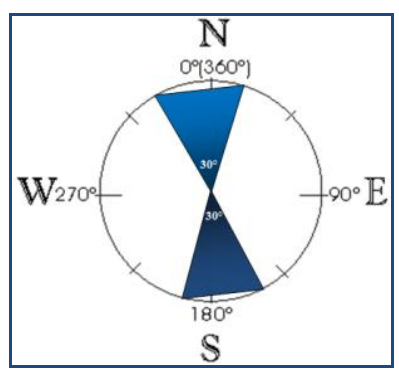

Figure 4. The assumed minimum view range of movement detection, i.e. $30^{\circ}$ in pro et contra flight direction for ascending pass as an example.
The slope aspect map of the study area has been used to identify slopes close to the satellite flight directions, and classify the entire area with respect to the satellite look direction.

\subsubsection{Upwards motion}

Some active surface deformation processes related to endogenous processes (such as tectonic and volcanic activity) may result in the upward surface motions. However, such types of motions are not the interest of this project. Thus, these types of motions can be identified by carefully analyzing the SAR acquisition geometry and the landslide motion direction.

Thus, for slope facing east, non-landslide or upwards moving pixels or DPs are filtered out from the GRID-DP as follows (refer section Error! Reference source not found. for visualization of variables or symbols used in this section and further details on the topic):

- Ascending orbit, DPs $>\mathbf{0 . 0} \mathrm{mm}$ represent upward motion. In fact, if the local slope angle $(\mathbf{u})$ is greater than the depression angle $(\boldsymbol{\alpha})$ of SAR sensor, i.e. $\boldsymbol{\alpha}$ $>\mathbf{6 2}^{\circ}$, then DPs are in shadow region.

- Descending orbit, DPs are affected by layover and/or foreshortening, and their effects. That is, if the local slope angle is greater than the look angle, $\mathbf{u}>\mathbf{2 8 ^ { \circ }}$, then DPs are affected by layover or its effect, and conversely, if $\mathbf{u}<\mathbf{2 8 ^ { \circ }}$, then DPs fall in foreshortening zones.

\section{For slope facing west,}

- Ascending orbit, DPs are affected by layover and/or foreshortening, and their effects.

- Descending orbit, DPs $>\mathbf{0 . 0} \mathrm{mm}$ represent upward motion. For the same reason, if $\boldsymbol{\alpha}>\mathbf{6 2}^{\circ}$, then DPs are affected by shadow effect.

\subsubsection{Downwards motion}

Landslide candidate DPs are finally filtered out using this parameter. By applying a similar approach like in the case of 
upwards motion, it is easy to filter out the landslide or downslope motion representing DPs from the GRID-DP.

\section{For slope facing east,}

- Ascending orbit, DPs $<\mathbf{0 . 0} \mathrm{mm}$ represent downwards or landslide motion. The depression angle $(\boldsymbol{\alpha})$ for ascending orbit was $65^{\circ}$. That is, if $\boldsymbol{\alpha}>\mathbf{6 5}^{\circ}$, then DPs are in shadow region.

- Descending orbit, DPs are affected by layover and/or foreshortening. By the same fashion, if the local slope angle is greater than the look angle, $\mathbf{u}>\mathbf{2 5}^{\circ}$, then DPs are affected by layover or its effect, and conversely, if $\mathbf{u}<\mathbf{2 5 ^ { \circ }}$, then DPs fall in foreshortening zones.

For slope facing west,

- $\quad$ Ascending orbit, DPs are affected by layover and/or foreshortening.

- Descending orbit, $\mathbf{D P s}<\mathbf{0 . 0} \mathrm{mm}$ represent downwards or landslide motion.

\subsubsection{Error DP}

It is common to experience data gaps in DInSAR analysis that are often associated with the use of external DEM data for topographic phase removal, and masking of low coherence pixels during phase unwrapping process. Though they are excluded from affecting the results of DInSAR analysis, the error pixels persist throughout the process. The masked areas are represented in the resulting displacement map with the value of 0.0 .

\subsubsection{GIS-MCFA analysis summary}

Each of the multiple criteria considered in the GIS-MCFA analysis had a distinctive interest in identifying the potential landslide representative DPs. Some of the filtering criteria have been used to remove the different noise sources attributed to SAR data. For instance, the geometrically distorted pixels or DPs as a result of high topography in the study area were filtered out through filtering criteria like "layover/shadow" and "extremely fast motion". Others control the deformation phenomena in terms of the direction of motion and speed of motion. As illustrated in the summary statistics Error! Reference source not found., and comparison bar diagram, Figure 3-2, each individual filtering criterion played its own significant role in filtering or excluding these error sources, and DPs affected by unwanted deformation signals from the grid.

All of the filtering criteria were equally applied for both ascending and descending radar data. However, the number of DPs affected in each of the acquisition geometries was variable. For example, if we consider the first criterion considered, i.e. "shadow and layover", the number of DPs affected by this criterion in both acquisition geometries were relatively high, and the number in descending excel more than twice than in ascending. Only a few number of DPs (less than 30,000 DPs in both ascending and descending data) were removed by applying some criteria like "extremely fast motion". On the contrary, much of the DPs were excluded from GRID using filtering criteria like "extremely slow motion", "geology and geomorphology", and "excluded slopes". Because some slopes are facing east in descending and west in ascending flight directions, they are suffered from geometric distortions. Noisy/error and unwanted deformation DPs were removed from a GRID sequentially one after the other (Table 1.). After removing all the possible noisy and unwanted DPs, the total remaining potential landslide representative DPs were 21972 and 12811 in ascending and descending SAR acquisition geometries respectively. 


\begin{tabular}{|c|c|c|c|c|c|}
\hline$\#$ & $\begin{array}{l}\text { Filtering } \\
\text { Criteria }\end{array}$ & $\begin{array}{l}\text { Filtering value or } \\
\text { conditions }\end{array}$ & $\begin{array}{l}\text { The no. and \% of } \\
\text { DPs affected DPs } \\
\text { (ASC,DSC) }\end{array}$ & $\begin{array}{l}\text { The no. and } \% \text { of } \\
\text { DPs remaining DPs } \\
\text { (ASC,DSC) }\end{array}$ & Remark \\
\hline 1 & $\begin{array}{l}\text { Layover/ } \\
\text { shadow }\end{array}$ & $\begin{array}{l}\text { Layover/Shadow } \\
\text { areas }\end{array}$ & $\begin{array}{l}71638(10 \%) \\
206131(29 \%)\end{array}$ & $\begin{array}{l}640506(90 \%), \\
509013(71 \%)\end{array}$ & $\begin{array}{l}\text { Areas affected by } \\
\text { overlay/shadow }\end{array}$ \\
\hline 2 & Coherence & $<0.35$ & $\begin{array}{l}93535(13.2 \%) \\
55617(8.4 \%)\end{array}$ & $\begin{array}{l}544971(76.8 \%), \\
444496(62.6)\end{array}$ & $\begin{array}{l}\text { Low coherent } \\
\text { areas }\end{array}$ \\
\hline 3 & $\begin{array}{l}\text { Geology and } \\
\text { geomorphology }\end{array}$ & $\begin{array}{l}\text { Pre-and post-rift } \\
\text { flood basalts }\end{array}$ & $\begin{array}{l}96855(13.6 \%), \\
101724(14.3 \%)\end{array}$ & $\begin{array}{l}448116(63.1 \%), \\
342772(48.3 \%)\end{array}$ & Stable areas \\
\hline 4 & $\begin{array}{l}\text { Extremely fast } \\
\text { motion }\end{array}$ & Slope & $\begin{array}{l}22144(2.8 \%), \\
6875(0.96 \%)\end{array}$ & $\begin{array}{l}427972(60.3 \%), \\
353897(47.3 \%)\end{array}$ & $\begin{array}{l}\text { Area of extremely } \\
\text { fast moving } \\
\text { landslides }\end{array}$ \\
\hline 5 & $\begin{array}{l}\text { Extremelyslow } \\
\text { motion }\end{array}$ & $\begin{array}{l}\text { DPs }<1 \mathrm{~mm} \\
\text { AND DPs }>-1 \mathrm{~mm}\end{array}$ & $\begin{array}{l}124572(17.5 \%), \\
36232(5.1 \%)\end{array}$ & $\begin{array}{l}303400(42.7 \%), \\
299665(42.2 \%)\end{array}$ & $\begin{array}{l}\text { No or extremely } \\
\text { slow movement } \\
\text { areas }\end{array}$ \\
\hline 6 & Non-los motion & $\begin{array}{l}30^{\circ} \text { in the azimuth } \\
\text { dir. }\end{array}$ & $\begin{array}{l}65263(9.2 \%), \\
41596(5.9 \%)\end{array}$ & $\begin{array}{c}238137(33.5 \%), \\
258069(36.3 \%)\end{array}$ & $\begin{array}{l}\text { Non-detectable } \\
\text { motions by SAR }\end{array}$ \\
\hline 7 & Upwardsmotion & $>0.0 \mathrm{~mm}$ & $\begin{array}{l}80753(11.4 \%)_{n} \\
39889(5.6 \%)\end{array}$ & $\begin{array}{l}157384(22.2 \%), \\
218180(30.7 \%)\end{array}$ & $\begin{array}{l}\text { Non-landslide } \\
\text { motions }\end{array}$ \\
\hline 8 & $\begin{array}{l}\text { Downwards } \\
\text { motion }\end{array}$ & $<0.0 \mathrm{~mm}$ & $\begin{array}{l}21972(3 \%), \\
12811(1.8 \%)\end{array}$ & $\begin{array}{l}135412(19.1 \%), \\
205369(28.9 \%)\end{array}$ & Landslidemotions \\
\hline 9 & $\begin{array}{l}\text { Slope excluded, } \\
\text { (ASCwast and } \\
\text { DSC:ant) }\end{array}$ & $\begin{array}{l}\text { Slope facing East = } \\
0<\text { Aspect }<180^{\circ} \& \\
\text { Slope facing West }= \\
180<\text { Aspect }<360^{\circ}\end{array}$ & $\begin{array}{l}126760(17.8 \%), \\
196060(27.6 \%)\end{array}$ & $\begin{array}{l}8652(1.2 \%), \\
9309(1.3 \%)\end{array}$ & $\begin{array}{l}\text { Slope facing EAST } \\
\text { (in DSC) \& WEST } \\
\text { (in ASC) data are } \\
\text { geometric errors }\end{array}$ \\
\hline 10 & Error & $D P_{s}=0.0$ & $\begin{array}{l}8652(1.2 \%), \\
9309(1.3 \%)\end{array}$ & 0,0 & $\begin{array}{l}\text { Data gaps in } \\
\text { DInSAR }\end{array}$ \\
\hline
\end{tabular}

Table 1. Summary of the GIS-MCFA analysis

The use of both ASC and DSC data enhanced the coverage of landslide detection, as ascending is suitable for slope facing towards to east (eastern slopes) and descending for western slopes (Notti et al. 2010, Colesanti et al. 2006). Using the two applied acquisition geometries in Dessie, it was possible to detect around $32 \%$ of landslide in ascending and $12.5 \%$ of landslide in descending orbits. The application of ASC and DSC data enabled the investigation of the entire area of interest.

The nature of landslide motion (speed and direction) has also controlled motion of a given landslide body to be along LOS and the rate of displacement not to exceed the detectable rage of the SAR sensor. The exposed steep slope areas and built-up sections of the city have revealed high coherent DPs. However, filtering criteria that control speed of motion ("extremely fast motion" and "extremely slow motion") have constrained these DPs as the target landslide bodies are set to be slow moving landslides in unstable sites. Consequently, areas of extremely fast slope movements (slopes greater than $45^{\circ}$ ) around steep slopes and stable areas in the city center were excluded from analysis.

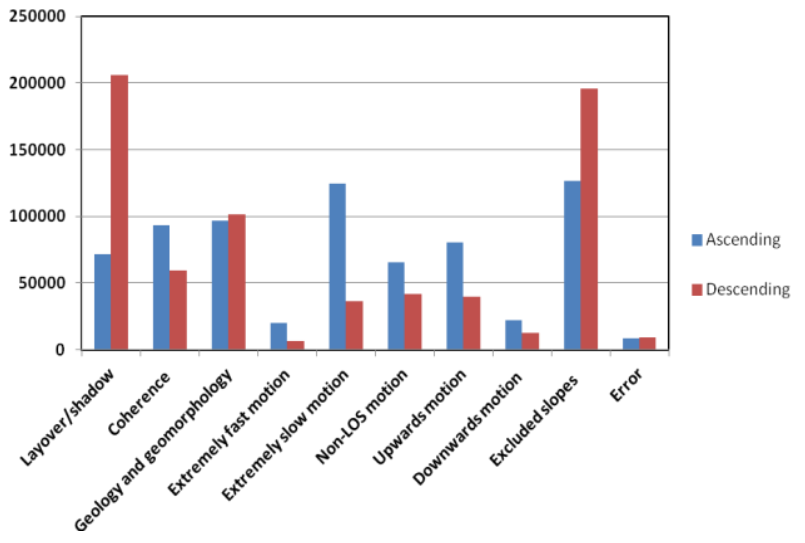

Figure 3-2: Compares the DPs filtering criteria in ascending and descending orbits.

Figure

from filtering out landslide representative DPs and mapping of landslide sites, the GIS-MCFA analysis helped to conduct a pixel based classification of the entire study area. This enables the study of specific target landslide bodies at their own original resolution and accurate geographic location, and without the need of generalization for the sake of simplicity. Based on the selected filtering criteria, the entire study area was globally classified as layover/shadow affected or not affected, low coherence or high coherence, extremely fast or slow moving landslide, stable or non-stable areas etc.

\subsection{Results of GIS-MCFA}

In the study area, the use of the GIS-MCFA and associated time-series curves indicated an increase in displacement during rainy months. April, July, and September were especially identified with high potential of slope failures. The LOS displacements recorded for a period of 11 months were in the range of $-30 \mathrm{~mm}$ to $+10 \mathrm{~mm}$.

Finally, the GRID-DP model might be of a choice for monitoring of slow moving landslides in mountainous terrains if there is no enough number of scenes available for PS-InSAR analysis, and want to integrate the radar data with external data sources and variables for specific purpose analysis.

\subsection{Reference}

Ayenew, T. \& Barbieri, G. (2005). Inventory of landslides and susceptibility mapping in the Dessie area, northern Ethiopia. Engineering Geology, 77(1), 1-15. 
Colesanti, C. \& Wasowski, J. (2006). Investigating landslides with space-borne Synthetic Aperture Radar (SAR) interferometry. Engineering geology, 88(3), 173-199.

Ferretti, A., Monti-Guarnieri, A., Prati, C., Rocca, F., Massonet, D. (2007).InSAR Principles-Guidelines for SAR Interferometry Processing and Interpretation (Vol. 19). ESA Publications.

Ferretti, A., Perissin, D., Prati, C., \& Rocca, F. (2005, April). On the physical nature of SAR Permanent Scatterers. In: Proceedings of URSI Commission Symposium Microwave Remote Sensing of the Earth, Oceans, Ice, and Atmosphere, Ispra, Italy.

Fubelli G., Abebe Bekele, Dramis F., Vinci S. (2008). Geomorphological evolution and present-day processes in the Dessie Graben (Wollo, Ethiopia). Catena, 75, 28-37.

Massonnet, D. \& Feigl, K. L. (1998). Radar interferometry and its application to changes in the Earth's surface. Reviews of geophysics, 36(4), 441-500.

Metternicht, G., Hurni, L. \& Gogu, R. (2005). Remote sensing of landslides: An analysis of the potential contribution to geo-spatial systems for hazard assessment in mountainous environments. Remote Sensing of Environment, 98, 284303.

Notti, D., Davalillo, J. C., Herrera, G., \& Mora, O. (2010). Assessment of the performance of X-band satellite radar data for landslide mapping and monitoring: Upper Tena Valley case study. Natural Hazards and Earth System Sciences, 10, 1865-1875.

Singhroy V. (2005). Remote Sensing for Landslide Assessment: Chapter 16. In: Landslides Hazard and Risk, book edited by Glade Anderson and Crozier. Wiley Press. pp. 469-492.

Acknowledgement: DLR is highly acknowledge for the supply of TSX/TDX data. 